\title{
Gibt es keine Alternativtherapie, muss die Kasse zahlen
}

\author{
Gerade bei seltenen Erkrankungen sind Ärzte unsicher, ob die Krankenkasse die \\ Therapiekosten nun übernimmt oder nicht, zumal es sich bei den Kosten oft nicht um \\ Bagatellbeträge handelt. Es gilt also, einen Regress unter allen Umständen zu vermeiden. \\ Doch dabei sind Ärzten die Hände gar nicht so stark gebunden, wie oft vermutet.
}

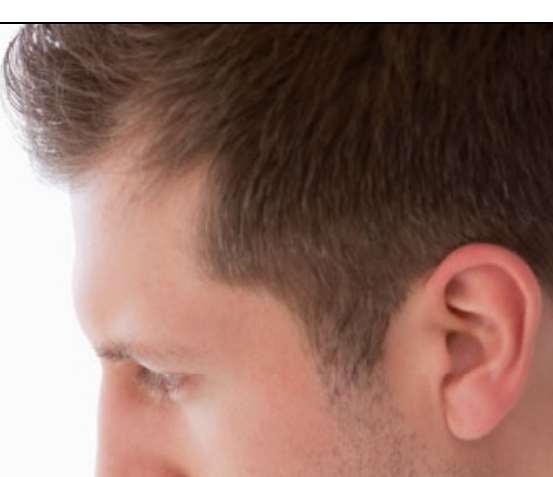

mmer wieder kommt es wegen teurer
Arzneien zu Konflikten zwischen Patienten, Krankenkassen und Ärzten. Bei singulären Therapien ist die Wirtschaftlichkeit allerdings mit der Zulassung gegeben, daher ist die Angst vor Regressen unbegründet. Das zeigt ein Fall zur Behandlung von Mucopolysaccharidose VI, einer sehr seltenen lysosomalen Speicherkrankheit. In Deutschland leidet durchschnittlich ein Neugeborenes pro Jahr darunter. Zur Behandlung ist seit 2006 eine Enzymersatztherapie, Naglazyme ${ }^{\circledast}$ (Galsulfase), zugelassen. Die Jahrestherapiekosten erreichen sechsstellige Beträge, bei erwachsenen Patienten auch eine Million Euro und mehr. Gerade für kleine Krankenkassen sind das erhebliche Kosten, die aufgrund fehlender Genauigkeit des Morbi-RSA nur zu einem geringen Teil über Zuweisungen aus dem Gesundheitsfonds refinanziert werden können.

\section{Der Fall}

Der zum Zeitpunkt der Zulassung besagter Enzymersatztherapie bereits deutlich über 30-jährige Patient leidet an einer vergleichsweise milden Verlaufsform der Mucopolysaccharidose. Trotzdem zeigt er bereits krankheitstypische Symptome wie multiple Kontrakturen, Herzklappenveränderungen und Hornhauttrübung. Zudem hatte er mehrfache Hüftgelenksendoprothesen. Für seinen betreuenden Arzt, einen Stoffwechselspezialisten, war die Indikation zur Behandlung mit Galsulfase offensichtlich. Allerdings verlangte die Klinikleitung eine Absicherung bei der
Krankenkasse. Den im Februar 2007 gestellten Kostenübernahmeantrag lehnte die Krankenkasse ab. Sie argumentierte, dass in die Zulassungsstudien nur Patienten im Alter zwischen 5 und 29 Jahren einbezogen waren und damit keine hinreichende Evidenz für die Wirksamkeit bei einem über 30-jährigen Patienten vorliege. Erst im Sommer 2011 kam es zu einer Entscheidung. Dabei gab es zwar keinen Zweifel an der Leistungspflicht der Krankenkasse, der Richter aber wollte die Therapie zwecks Evaluation zunächst auf drei Monate begrenzen (Sozialgericht Münster - S 16 (11) KR 78/09). Da dies schon deswegen nicht sinnvoll war, weil eine Enzymersatztherapie nicht binnen dreier Monate belastbare Ergebnisse zur individuellen Wirksamkeit ergeben kann, wurde eine Berufung zum Landessozialgericht Nordrhein-Westfalen erforderlich (L 5 KR 601/11).

In der mündlichen Verhandlung im September 2012 wurden die LSG-Richter deutlich: Es sei völlig unverständlich, dass ein Patient, der ganz offensichtlich dem zugelassenen Anwendungsgebiet der einzig verfügbaren Therapie unterfalle, mehr als fünf Jahre unbehandelt bleibe, weil sich die Ärzte nicht trauten, die offensichtlich rechtmäßige Verordnung auf Kassenrezept vorzunehmen und weil die Krankenkasse mit offensichtlich unbegründeten Argumenten den Behandlungsbeginn hinauszögere. Die Richter deuteten an, dass der Patient möglicherweise einen Schadensersatzanspruch zum Ausgleich in der Zwischenzeit entstandener weiterer Gesundheitsschäden habe. Die
Krankenkasse sagte daraufhin die Kostenübernahme zu.

\section{Die Rechtslage}

Gemäß der $₫ \$ 2,12,27,31,34$ SGB V haben GKV-Patienten Anspruch auf Arzneimittel, die nicht aus der Versorgung ausgeschlossen sind, sofern die Behandlung dem allgemein anerkannten Stand der medizinischen Erkenntnis und dem Wirtschaftlichkeitsgebot entspricht. Jedenfalls dann, wenn eine Arzneimitteltherapie alternativlos ist, entspricht sie mit der Zulassung dem medizinischen Stand und ist im Sinne des Wirtschaftlichkeitsgebots zweckmäßig sowie notwendig. Auch das Wirtschaftlichkeitsgebot im engeren Sinne steht der Verordnung teurer Therapien nur dann entgegen, wenn das angestrebte Therapieziel kostengünstiger erreicht werden kann. Besteht indessen ein medizinischer Anlass zur Verordnung einer teuren Therapie, ist diese auch wirtschaftlich (Bundessozialgericht - B 6 KA 41/03). Die Abwägung des Kosten-Nutzen-Verhältnisses einer Therapie gehört nicht zu den Aufgaben des verordnenden Arztes (Bundessozialgericht - B 6 KA 13/05 R). Hieraus folgt: Da der Patient dem zugelassenen Anwendungsgebiet von Naglazyme ${ }^{\circledast}$ unterfiel, hatte er einen Anspruch darauf. Das Argument, die Zulassungsstudien ergäben keine Evidenz für Patienten jenseits des 30. Lebensjahres mit milderen Verlaufsformen, überzeugt nicht. Es ist Aufgabe der Zulassungsbehörde das Anwendungsgebiet abzustecken. Die Krankenkasse darf eine Begrenzung ihrer Leistungspflicht nicht in die Zulassung hineinlesen.

Dr. Gerhard Nitz 